\title{
Neues aus der Arbeitsgruppe WZW und zu den Labortarifen
}

\section{Ernst Gählera,}

Thomas Kessler ${ }^{b}$,

Susanne Christen ${ }^{c}$

a Dr. med., Vizepräsident FMH, Verantwortlicher Ressort Ambulante Tarife und Verträge Schweiz

b FMH, Ressort Ambulante Tarife und Verträge Schweiz

c Dr. med., FMH, Ressort Ambulante Tarife und Verträge Schweiz
Korrespondenz:

FMH / Ressort Ambulante Tarife und Verträge Schweiz

Froburgstrasse 15

CH-4600 Olten

Tel. 0313591230

Fax 0313591238

tarife.ambulant[at]fmh.ch

\section{Arbeitsgruppe WZW - \\ Aktueller Stand der Verhandlungen}

An der Sitzung der Arbeitsgruppe WZW (Wirksamkeit, Zweckmässigkeit, Wirtschaftlichkeit) vom 27. November 2013 einigten sich die Vertragsparteien (FMH, santésuisse und curafutura) auf einen gemeinsamen Vertrag zu Art. 56 Abs. 6 des Krankenversicherungsgesetzes. Dieser Artikel sieht vor, dass «Leistungserbringer und Versicherer vertraglich eine Methode zur Kontrolle der Wirtschaftlichkeit festlegen». Der Vertrag, der dem Bundesrat zur Kenntnisnahme unterbreitet wird, wurde durch die Vertragsparteien gemäss den gesetzlichen Vorgaben erstellt und enthält die folgenden vier zentralen Punkte:

1. Als statistische Methode zur Kontrolle der Wirtschaftlichkeit wird die Varianzanalyse festgelegt. Die Varianzanalyse wird zur Beurteilung von beobachteten Unterschieden zwischen Leistungserbringern innerhalb ihrer Vergleichsgruppe in den Wirtschaftlichkeitsverfahren verwendet.

2. Das heute verwendete Varianzanalysemodell mit demographischen, erklärenden Variablen soll künftig von Leistungserbringern und Versicherern gemeinsam weiterentwickelt und unter anderem durch Morbiditätsvariablen ergänzt werden.

3. Die erklärenden Variablen sollen auf der Basis einer stetig sich verbessernden Datengrundlage in neuen statistischen Modellen erprobt und validiert werden.

4. Neben dem Auftrag des Gesetzgebers, die statistische Methode festzulegen, vereinbaren die Leistungserbringer und die Krankenversicherer, die Kontrolle der Wirtschaftlichkeit durch die paritätischen Vertrauenskommissionen (PVK) zu vereinheitlichen und $\mathrm{zu}$ professionalisieren. Die Regelung dieser Vereinheitlichung und Professionalisierung wird in einer gemeinsamen Vereinbarung festgelegt.

Im Rahmen der Arbeitsgruppe WZW arbeiten aktuell drei Subgruppen, die spezifische Themenbereiche behandeln und entsprechende Lösungen aufzeigen:

- Subgruppe 1 «ANOVA»: Verfolgt das Ziel, die statistischen Methoden (Durchschnittskosten-Methode und Varianzanalyse) dahingehend zu verbessern, dass weniger falsch positive und weniger falsch negative Leistungserbringer im Rahmen der Wirtschaftlichkeitskontrolle erfasst werden. Es werden facharztgruppenspezifisch diejenigen Variablen für die Varianzanalyse ermittelt, welche die Kosten der Leistungserbringung durch den Arzt in seinem Vergleichskollektiv empirisch valide erklären können. Die entsprechenden Arbeiten sind am Laufen.

- Subgruppe 2 «PV»: Die Abläufe und Strukturen der kantonalen und regionalen paritätischen Vertrauenskommissionen sollen vereinheitlicht und professionalisiert werden. Zukünftig gibt es voraussichtlich nur noch drei regionale PVKs (Deutsch- bzw. Westschweiz sowie Tessin). Das Reglement der PVKs befindet sich zurzeit in Vernehmlassung bei den Vertragsparteien.

- Subgruppe 3 «Homogenisierung der Vergleichskollektive»: Verfolgt das gleiche Ziel wie die Subgruppe 1 «ANOVA». Das Ziel soll hier mit Hilfe einer verfeinerten Bildung von Vergleichskollektiven auf Basis einer verbesserten Datengrundlage und durch Berücksichtigung von Morbiditätsfaktoren erreicht werden. Die verfeinerte Bildung von Vergleichskollektiven erfolgt unter Beizug der medizinischen Fachgesellschaften und versteht sich im Sinne eines lernenden Systems. Die entsprechenden Arbeiten sind am Laufen.

Zudem erarbeiten die Vertragsparteien einen Rahmenvertrag, der die detaillierten Modalitäten der Wirtschaftlichkeitskontrolle aufzeigt und in Form einer Roadmap definiert, welche Arbeiten bis wann abgeschlossen sein müssen. Die nächste Sitzung der Arbeitsgruppe WZW findet am 22.1.2014 statt.

\section{Labor - \\ Entscheidungen beim Point-of-Care-Tarif}

Bundesrat Alain Berset hat - aufgrund der faktenbasierten Positionierung der FMH - entschieden, dass der Point-of-Care-Tarif (POCT) 33 «Schnelle Analysen» beinhalten wird. Ebenso hat er aufgrund der Monitoring-Resultate entschieden, die $\mathrm{zu}$ starke Absenkung im Praxislabor mit 35 Millionen Franken zu kompensieren. Zur Bewertung der 33 Leistungen beim POCT kommt erfreulicherweise das Kostenmodell der FMH zur Anwendung. Offene Fragen im Kostenmodell sind der Einbezug und die Wertigkeit der indirekten Kosten. Zudem gab es noch offene Fragen betreffend Abrechnungsberichtigung. Folgende Entscheidungen sind diesbezüglich nun getroffen:

- Die Apotheken werden den neuen Tarif der «schnellen Analysen» nicht abrechnen dürfen. - Die Positionen des Kapitels «schnelle Analysen» 
können von allen Praxislaboratorien gemäss Art. 54 Abs. 1 lit. a Krankenversicherungsverordnung abgerechnet werden. Das heisst, sowohl von den Grundversorgern (Allgemeine Innere Medizin, Kinder und Jugendmedizin, praktische Ärzte gemäss Medizinalberufeverordnung, Anhang 1) als auch von den Spezialärzten.

\section{Die zu starke Absenkung im Praxislabor wird mit 35 Millionen Franken kompensiert.}

Offen ist noch, ab welchem Zeitpunkt die POCTTarifstruktur zur Anwendung kommen wird.

\section{Labor - Übergangstaxpunkt}

Da die POCT-Tarifstruktur per 1.1.2014 noch nicht zur Anwendung kommen kann, wird der Übergangstaxpunkt - aktuell 1.1 Taxpunkte - ab 1. Januar 2014 bis zum Inkrafttreten des POCT auf 1.9 TP = 1.90 CHF angehoben, damit die 35 Millionen Franken der Ärzteschaft bereits ab dem Jahreswechsel zur Verfügung stehen.

\section{Labor - QUALAB - Neue Toleranzgrenzwerte ab 1. Januar 2014}

In der QUALAB wurden einige ToleranzgrenzwertAnpassungen der internen (IQK) und externen Qualitätskontrolle (EQK) vorgenommen. Es betrifft folgende Parameter: Blutgase: $\mathrm{pH}$ und $\mathrm{pCO}_{2}$, LaktatDehydrogenase (LDH), Harnsäure, Vitamin D3 bzw. 25-Hydroxycholecalciferol. Die neuen Toleranzgrenzwerte sind ab dem 1. Januar 2014 gültig.

Die Richtlinien und Listen zur internen Qualitätskontrolle sowie der externen obligatorischen Qualitätskontrolle 2014 finden Sie unter www.qualab.ch.

\section{Wissen, was läuft. Das News-Paket der FMH.}

Schweizerische Ärztezeitung, Today's Press, FMH-Flash. Für Mitglieder kostenlos. 